\title{
Iterative roots of upper semicontinuous multifunctions
}

\author{
Pingping Zhang* and Liguo Huang
}

"Correspondence: zhangpingpingmath@163.com Department of Mathematics, Binzhou University, Shandong, 256603, P.R. China

\begin{abstract}
The square iterative roots for strictly monotonic and upper semicontinuous functions with one set-valued point were fully described in (Li et al. in Publ. Math. (Debr.) 75:203-220, 2009). As a continuation, we study both strictly monotonic and nonmonotonic multifunctions. We present sufficient and necessary conditions under which those multifunctions have $n$th iterative roots. This equivalent condition and the construction method of $n$th iterative roots extend the previous results.
\end{abstract}

MSC: 39B12; 37E05

Keywords: iterative root; one-to-one; upper semicontinuous multifunction; set-valued point

\section{Introduction}

Given a mapping $F: X \rightarrow X$ and an integer $n \geq 2$, the iterative root problem is to find all self-mappings $f: X \rightarrow X$ such that their $n$th iterates satisfy the functional equation

$$
f^{n}=F
$$

Babbage [2] investigated (1.1) for an identity mapping $F$ as far back as the 1810s. After that, (1.1) has been studied in various aspects and settings since it is an important subject in the theory of functional equations; we refer to the survey papers [3-7], the monographs $[8,9]$, and the book [10]. For all we know, strictly increasing roots of strictly increasing and continuous functions were discussed by Bödewadt [11], and their strictly decreasing roots were presented by Haìdukov [12]. In 1961, Kuczma [13] gave a complete description of strictly monotonic and continuous functions having roots. However, even simple nonmonotonic functions can have no iterative roots, for example, the hat functions $f(x)=\min \left\{\frac{x}{a}, \frac{1-x}{1-a}\right\}$ on the compact interval $[0,1]$ for arbitrary $a \in(0,1)$. In 1983, Zhang and Yang [14] investigated the roots of piecewise monotonic functions (abbreviated as PM functions). The main difficulties to find roots of PM functions lie in the continuously increasing number of nonmonotonic points under iteration (see [15]). Their method is based on the 'characteristic interval', which was developed in $[7,16]$. In recent years, many important results on iterative roots of PM functions were presented in [17-19]. It is worth mentioning that those results are related to single-valued functions. In [20, 21] and [22], it is illustrated that the set of continuous functions having a root is a non-Borel subset of 
$C([0,1], \mathbb{R})$ and is small in $C([0,1],[0,1])$. That is to say, in the general case, no such roots exist, and the theory becomes extremely complicated if $F$ is not bijective [23]. Therefore, it is a natural idea to extend the notion of iterative root.

In his survey paper [5], Targonski illustrated three ways to generalize iterative roots, extending or restricting the domain of the function or embedding the semigroup of selfmappings in a larger semigroup, and discussed the so-called phantom iterative root of continuous functions in [23]. Powierża and Jarczyk [24-26] gave set-valued functions as roots of single-valued functions. Maybe the best method to generalize iterative roots is replacing single-valued functions by set-valued functions for both $F$ and $f$ in (1.1) (see [4]). It seems that, up to now, there are only several results on set-valued iterative roots of multifunctions, even with a unique set-valued point. In 2007, Jarczyk and Zhang [27] considered the nonexistence of square iterative roots of multifunctions with exactly one set-value point and presented two sufficient conditions for the purely set-theoretical situation. Later, Li, Jarczyk, Jarczyk, and Zhang [1] gave new nonexistence results for purely set-theoretical case and fully described the square roots of strictly monotonic, upper semicontinuous (abbreviated as usc) multifunctions.

As a continuation of [1], in this paper, we study all strictly monotonic usc multifunctions having one set-valued point and partly nonmonotonic ones. We give sufficient and necessary conditions for the existence of $n$th iterative roots and their construction method, which extend the results on strictly monotonic usc multifunctions in [1]. In Section 2, we recall the basic definitions and present Lemmas 1-3. In Section 3, we give equivalent conditions for the existence of $n$th iterative roots and their expressions. Finally, in Section 4, we apply examples to illustrate our results.

\section{Preliminaries}

Given topological spaces $X$ and $Y$, a multifunction $f: X \rightarrow 2^{Y}$ is called upper semicontinuous at a point $x_{0} \in X$ if for every open set $V \subset Y$ with $f\left(x_{0}\right) \subset V$, there exists a neighborhood $U \subset X$ of $x_{0}$ such that $f(U) \subset V$. If $f$ is upper semicontinuous at every point of $B \subset X$, then it is called upper semicontinuous on a set $B \subset X$. Let $F: X \rightarrow X$ and $G: Y \rightarrow Y$ be continuous functions. We say that $F$ is topologically conjugate to $G$ if there exists a homeomorphism $\varphi: X \rightarrow Y$ satisfying the equation $\varphi \circ F=G \circ \varphi$.

Let $X$ be an interval $I:=[a, b] \subset \mathbb{R}$. For a multifunction $f: I \rightarrow 2^{I}$, the image $f(A)$ for a set $A \subset I$ is defined by $f(A):=\bigcup_{x \in A} f(x)$. For a given positive integer $n$, we define the $n$th iterate of $f: I \rightarrow 2^{I}$ as the composition of $n$ copies of $f$ :

$$
f^{n}(x):=\bigcup_{y \in f^{n-1}(x)} f(y)
$$

where $f^{0}(x):=\{x\}$ for every $x \in I$. Let $\# A$ denote the cardinality of the set $A$. Then a point $x_{0} \in(a, b)$ is said to be a set-valued point of $f$ if the cardinality $\# f\left(x_{0}\right) \geq 2$. Denote by $\mathcal{S}_{x_{0}}(I, I)$ the set of all multifunctions $f: I \rightarrow 2^{I}$ that are continuous on $I$ except for the single set-valued point $x_{0}$. We say that $f$ is one-to-one on $I$ if $f\left(x_{1}\right) \neq f\left(x_{2}\right)$ for all different $x_{1}, x_{2} \in I \backslash\left\{x_{0}\right\}$. 
Definition 1 ([1]) Let $\gamma: I \rightarrow I$ be a strictly increasing continuous function. Given a fixed point $\xi \in I$ of $\gamma$, we put

$$
A_{\xi}=\{x \in \operatorname{cl} I: \gamma(x)=x \leq \xi\}, \quad B_{\xi}=\{x \in \operatorname{cl} I: \gamma(x)=x \geq \xi\} .
$$

Then $\xi$ is called a regular fixed point of $\gamma$ if

(a) $\min A_{\xi}=\inf I$ if and only if $\max B_{\xi}=\sup I$;

(b) there exists a strictly decreasing function $a$ mapping $A_{\xi}$ onto $B_{\xi}$;

(c) for every component $(a, b)$ of the set $\{x \in \operatorname{cl} I: \gamma(x) \neq x\}$, where $a \in A_{\xi} \cup\{\inf I\}$, $b \in A_{\xi}$, and $a<b$, the graphs of $\left.\gamma\right|_{(a, b)}$ and $\left.\gamma\right|_{(\alpha(a), \alpha(b))}$ lie on the opposite sides of the diagonal.

Definition 2 ([1]) A multifunction $f: I \rightarrow 2^{I}$ is called strictly increasing (strictly decreasing) if $\sup f\left(x_{1}\right)<\inf f\left(x_{2}\right)\left(\inf f\left(x_{1}\right)<\sup f\left(x_{2}\right)\right)$ whenever $x_{1}, x_{2} \in I$ and $x_{1}<x_{2}$. Multifunctions that are either strictly increasing or strictly decreasing are called strictly monotonic.

It is clear that every strictly monotonic multifunction is one-to-one. Conversely, a oneto-one multifunction is not necessarily strictly monotonic. In this paper, we investigate the $n$th iterative roots of usc multifunctions $F \in \mathcal{S}_{x_{0}}(I, I)$ of the form

$$
F(x)= \begin{cases}\left.F\right|_{L_{-}}, & x \in I_{-}:=\left[a, x_{0}\right), \\ {[c, d]([d, c]),} & x=x_{0}, \\ \left.F\right|_{I_{+}}, & x \in I_{+}:=\left(x_{0}, b\right],\end{cases}
$$

where $[c, d]([d, c]) \subset I$, and $\left.F\right|_{I_{-}}$and $\left.F\right|_{I_{+}}$are strictly monotonic and satisfy

$$
\lim _{x \rightarrow x_{0}^{-}} F(x)=c, \quad \lim _{x \rightarrow x_{0}} F(x)=d .
$$

The next lemmas describe the fundamental properties of the $n$th iterative roots of (2.1).

Lemma 1 If $F \in \mathcal{S}_{x_{0}}(I, I)$ is one-to-one, then every nth iterativeroot $f$ of $F$ belongs to $\mathcal{S}_{x_{0}}(I, I)$ and is also one-to-one.

Proof We first prove that $f$ is one-to-one. Suppose on the contrary that there exist two different points $u, v \in I$ such that $f(u)=f(v)$. Then $f^{n}(u)=f^{n}(v)$, and thus $F(u)=F(v)$. Since $F$ is one-to-one, only the case $u=v$ is possible, contrary to the assumption.

Now we show that $f \in \mathcal{S}_{x_{0}}(I, I)$. For any $y \in I \backslash\left\{x_{0}\right\}$, suppose on the contrary that $\# f(y) \geq 2$. Then we have

$$
\# F(y)=\# f^{n}(y) \geq 2,
$$

which contradicts $F \in \mathcal{S}_{x_{0}}(I, I)$. Thus, $f(y)$ is a singleton for all $y \in I \backslash\left\{x_{0}\right\}$. We claim that $x_{0}$ is a unique set-valued point of $f$. Otherwise, let $f\left(x_{0}\right)=\left\{p_{0}\right\}$. Then two cases are possible: either $p_{0}=x_{0}$ or $p_{0} \neq x_{0}$. From the former we have

$$
F\left(x_{0}\right)=f^{n}\left(x_{0}\right)=x_{0},
$$


which contradicts $\# F\left(x_{0}\right) \geq 2$. For the case $p_{0} \neq x_{0}, f \in \mathcal{S}_{x_{0}}(I, I)$ implies that $\# f\left(p_{0}\right)=1$ since $f$ is single-valued at $I \backslash\left\{x_{0}\right\}$. Without loss of generality, assume that $f\left(p_{0}\right)=\left\{p_{1}\right\}$. Then there again exist two cases: either $p_{1}=x_{0}$ or $p_{1} \neq x_{0}$. If $p_{1}=x_{0}$, a contradiction comes from

$$
F\left(x_{0}\right)=f^{n-2}\left(p_{1}\right)=f^{n}\left(x_{0}\right)=\left\{x_{0}\right\} .
$$

If $p_{1} \neq x_{0}$, we have $\# f\left(p_{1}\right)=1$ since $f$ is single-valued except for $x_{0}$. Repeating this progress, we inductively obtain

$$
\# f\left(p_{n-2}\right)=1 \quad \text { for } p_{n-2} \neq x_{0}
$$

and, consequently, we have

$$
\# F\left(x_{0}\right)=\# f^{n}\left(x_{0}\right)=\# f\left(p_{n-2}\right)=1,
$$

which contradicts $F \in \mathcal{S}_{x_{0}}(I, I)$. Thus, we prove $f \in \mathcal{S}_{x_{0}}(I, I)$. This completes the proof.

Lemma 2 If $F \in \mathcal{S}_{x_{0}}(I, I)$ is one-to-one, then $F$ has no nth iterative root $f \in \mathcal{S}_{x_{0}}(I, I)$ taking the value $\left\{x_{0}\right\}$.

Proof Assume that $f$ is an $n$th iterative root of $F$. We claim that $f \in \mathcal{S}_{x_{0}}(I, I)$ by Lemma 1 . Suppose on the contrary that $f\left(y_{0}\right)=\left\{x_{0}\right\}$ for a point $y_{0} \in I \backslash\left\{x_{0}\right\}$. Then

$$
F\left(y_{0}\right)=f^{n}\left(y_{0}\right)=f^{n-1}\left(x_{0}\right),
$$

whence

$$
\# F\left(y_{0}\right)=\# f^{n-1}\left(x_{0}\right) \geq 2,
$$

implying $y_{0}=x_{0}$, a contradiction. This completes the proof.

Lemma 3 Suppose that $F \in \mathcal{S}_{x_{0}}(I, I)$ is one-to-one.

(i) If $F$ is strictly increasing, then $F$ has no strictly decreasing $n$th iterative root for odd $n$.

(ii) If $F$ is strictly decreasing, then $F$ has no strictly increasing $n$th iterative root for even $n$.

This proof is trivial and omitted.

\section{Main results}

In this section, we give several sufficient and necessary conditions and expressions of $n$th iterative roots of (2.1). Theorem 1 and Theorem 3 characterize the strictly monotonic usc multifunctions, and nonmonotonic cases are investigated in Theorem 2 and Theorem 4. For convenience, let $f_{1}:=\left.f\right|_{I_{-}}, f_{2}:=\left.f\right|_{I_{+}}$.

Theorem 1 Suppose that the usc multifunction (2.1) has $F\left(x_{0}\right)=[c, d]$. If $\left.F\right|_{I_{-}}$and $\left.F\right|_{I_{+}}$ are strictly increasing, then $F$ has nth iterative roots if and only if $F$ satisfies $F(b)<x_{0}$ or $F(a)>x_{0}$ or $c \leq x_{0} \leq d$. 
(i) If $F(b)<x_{0}$, then $F$ has a strictly increasing $n$th iterative root

$$
f(x)= \begin{cases}f_{1}(x), & x \in I_{-}, \\ f_{1}^{-(n-1)} \circ F(x), & x \in I_{+} \cup\left\{x_{0}\right\},\end{cases}
$$

where $f_{1}: I_{-} \rightarrow I_{-}$is a strictly increasing function satisfying $f_{1}{ }^{n}=\left.F\right|_{I_{-}}$. Moreover, if $n$ is even and $\left.F\right|_{I_{-}}$has a regular fixed point, then $F$ also has a strictly decreasing $n$th iterative root of the form (3.1), in which $f_{1}$ is a strictly decreasing function satisfying $f_{1}{ }^{n}=\left.F\right|_{I_{-}}$.

(ii) If $F(a)>x_{0}$, then $F$ has a strictly increasing nth iterative root

$$
f(x)= \begin{cases}f_{2}^{-(n-1)} \circ F(x), & x \in I_{-} \cup\left\{x_{0}\right\} \\ f_{2}(x), & x \in I_{+}\end{cases}
$$

where $f_{2}: I_{+} \rightarrow I_{+}$is a strictly increasing function satisfying $f_{2}{ }^{n}=\left.F\right|_{I_{+}}$. Moreover, if $n$ is even and $\left.F\right|_{I_{+}}$has a regular fixed point, then $F$ also has a strictly decreasing $n$th iterative root of the form (3.2), in which $f_{2}$ is a strictly decreasing function satisfying $f_{2}{ }^{n}=\left.F\right|_{I_{+}}$.

(iii) If $c \leq x_{0} \leq d$, then $F$ has a strictly increasing $n$th iterative root

$$
f(x)= \begin{cases}f_{1}(x), & x \in I_{-} \\ {\left[f_{1}^{-(n-1)}(c), f_{2}^{-(n-1)}(d)\right],} & x=x_{0} \\ f_{2}(x), & x \in I_{+}\end{cases}
$$

where $f_{1}$ and $f_{2}$ are defined as in (3.1) and (3.2), respectively. Moreover, if $n$ is even and $\left.F\right|_{I_{+}}$is topologically conjugate to $\left.F\right|_{I_{-}}$by a strictly decreasing function $f_{1}: I_{-} \rightarrow I_{+}$, that is, $\left.F\right|_{I_{+}}=\left.f_{1} \circ F\right|_{I_{-}} \circ f_{1}^{-1}$, then $F$ also has a strictly decreasing $n$th iterative root

$$
f(x)= \begin{cases}f_{1}(x), & x \in I_{-}, \\ {\left[\left.f_{1}^{-1} \circ g\right|_{I_{+}} \circ\left(\left.F\right|_{I_{+}}\right)^{-1}(d),\left.\left(\left.g\right|_{I_{+}}\right)^{-1} \circ f_{1} \circ g\right|_{I_{-}} \circ\left(\left.F\right|_{L_{-}}\right)^{-1}(c)\right],} & x=x_{0}, \\ \left.g\right|_{I_{-}} \circ f_{1}^{-1}(x), & x \in I_{+},\end{cases}
$$

in which $g$ is a strictly increasing $\frac{n}{2}$ th iterative root of $F$.

Proof Necessity. If $F$ has $n$th iterative roots, then $F$ does not take the value $\left\{x_{0}\right\}$ by Lemma 2 . Therefore, only the following cases are possible:

Case 1. $F(I) \subset I_{-}$, i.e., $F(b)<x_{0}$, or

Case 2. $F(I) \subset I_{+}$, i.e., $F(a)>x_{0}$, or

Case 3. $F\left(I_{-}\right) \subset I_{-}$and $F\left(I_{+}\right) \subset I_{+}$, i.e., $c \leq x_{0} \leq d$.

Sufficiency. Consider case (i). Observing that $\left.F\right|_{I_{-}}: I_{-} \rightarrow I_{-}$is strictly increasing, it follows from Theorem 11.2.2 in [9] that there exists a strictly increasing function $f_{1}: I_{-} \rightarrow I_{-}$ such that

$$
f_{1}^{n}=\left.F\right|_{I_{-}}
$$


For every $x \in I_{+} \cup\left\{x_{0}\right\}$, using that $F(x) \subset I_{-}$and $f_{1}$ is strictly increasing, from

$$
F(x)=f^{n-1}(f(x))=f_{1}{ }^{n-1}(f(x))
$$

we get

$$
f(x)=f_{1}^{-(n-1)} \circ F(x), \quad x \in I_{+} \cup\left\{x_{0}\right\} .
$$

Furthermore, by the upper semicontinuity property at the set-value point $x_{0}$ we have

$$
\begin{aligned}
& c=F\left(x_{0}{ }^{-}\right)=f_{1}{ }^{n-1} \circ f\left(x_{0}{ }^{-}\right), \\
& d=F\left(x_{0}{ }^{+}\right)=f_{1}{ }^{n-1} \circ f\left(x_{0}{ }^{+}\right) .
\end{aligned}
$$

Since $f_{1}{ }^{n-1}$ is strictly increasing, it follows from (3.8) that

$$
f\left(x_{0}{ }^{-}\right)<f\left(x_{0}{ }^{+}\right) .
$$

Hence, formulas (3.5), (3.7), and (3.9) give a strictly increasing $n$th iterative $\operatorname{root}(3.1)$ of $F$. Assuming that $n$ is even, the left end-point $a$ is not a fixed point of $\left.F\right|_{-}$since it has a regular fixed point, and there exists a strictly decreasing $n$th iterative $\operatorname{root} f_{1}: I_{-} \rightarrow I_{-}$of $\left.F\right|_{-}$(see [9, p.427]). Moreover, since $f_{1}$ is strictly decreasing, using (3.8), we have

$$
f\left(x_{0}^{-}\right)>f\left(x_{0}^{+}\right)
$$

Thus, $f_{1}$ and (3.7) and (3.10) give a strictly decreasing $n$th iterative root (3.1) of $F$.

The proof of case (ii) is immediately obtained by the transformation

$$
g(x):=b-a-f(x) \text { for } x \in I .
$$

Case (iii). The condition $c \leq x_{0} \leq d$ implies that $F\left(I_{-}\right) \subset I_{-}$and $F\left(I_{+}\right) \subset I_{+}$. Since $\left.F\right|_{I_{-}}$and $\left.F\right|_{I_{+}}$are strictly increasing, their strictly increasing $n$th iterative roots $f_{1}$ and $f_{2}$ exist and are defined as in (3.1) and (3.2), respectively. Observing that $f$ is one-to-one by Lemma 1 and $f_{1}$ and $f_{2}$ are strictly increasing, we have

$$
\begin{aligned}
& c=F\left(x_{0}{ }^{-}\right)=f^{n}\left(x_{0}{ }^{-}\right)=f^{n-1}\left(f\left(x_{0}{ }^{-}\right)\right)=f_{1}{ }^{n-1}\left(f\left(x_{0}{ }^{-}\right)\right), \\
& d=F\left(x_{0}{ }^{+}\right)=f^{n}\left(x_{0}{ }^{+}\right)=f^{n-1}\left(f\left(x_{0}{ }^{+}\right)\right)=f_{2}{ }^{n-1}\left(f\left(x_{0}{ }^{+}\right)\right),
\end{aligned}
$$

which yields (3.9). Consequently, $f_{1}$ and $f_{2}$ together with (3.12) and (3.9) give a strictly increasing $n$th iterative root (3.3) of $F$.

If $n$ is even and $\left.F\right|_{I_{+}}$is topologically conjugate to $\left.F\right|_{I_{-}}$by a strictly decreasing function $f_{1}: I_{-} \rightarrow I_{+}$, then we will prove that $F$ also has a strictly decreasing $n$th iterative $\operatorname{root} f$ of $F$. In fact, this problem reduces itself to a solution of the system

$$
\left\{\begin{array}{l}
f^{2}=g, \\
g^{\frac{n}{2}}=F .
\end{array}\right.
$$


As in the previous argument, $F$ possesses a strictly increasing $\frac{n}{2}$ th iterative root $g$, and we confine ourselves to the first equation $f^{2}=g$ to find a strictly decreasing solution $f$ of $F$. The given condition $\left.F\right|_{I_{+}}=\left.f_{1} \circ F\right|_{I_{-}} \circ f_{1}^{-1}$ implies that the strictly increasing functions $\left.g\right|_{I_{+}}$ and $\left.g\right|_{I_{-}}$satisfy the conjugacy equation

$$
\left.g\right|_{I_{+}}=\left.f_{1} \circ g\right|_{I_{-}} \circ f_{1}^{-1}
$$

and the equality $g^{\frac{n}{2}}=F$ shows that

$$
\left.g\right|_{I_{+}}=f_{1} \circ f_{2},\left.\quad g\right|_{I_{-}}=f_{2} \circ f_{1} .
$$

The second equality in (3.15) yields that

$$
f_{2}=\left.g\right|_{I_{-}} \circ f_{1}^{-1}
$$

implying that $f_{2}: I_{+} \rightarrow I_{-}$also is strictly decreasing. Moreover,

$$
\begin{aligned}
c & =F\left(x_{0}{ }^{-}\right) \\
& =g^{\frac{n}{2}}\left(x_{0}{ }^{-}\right) \\
& =\left(\left.g\right|_{I_{-}}\right)^{\frac{n}{2}-1} \circ f_{2} \circ f_{1}\left(x_{0}{ }^{-}\right) \\
& =\left.F\right|_{I_{-}} \circ\left(\left.g\right|_{I_{-}}\right)^{-1} \circ f_{2} \circ f\left(x_{0}^{-}\right), \\
d & =F\left(x_{0}^{+}\right) \\
& =g^{\frac{n}{2}}\left(x_{0}^{+}\right) \\
& =\left(\left.g\right|_{I_{+}}\right)^{\frac{n}{2}-1} \circ f_{1} \circ f_{2}\left(x_{0}^{+}\right) \\
& =\left.F\right|_{I_{+}} \circ\left(\left.g\right|_{I_{+}}\right)^{-1} \circ f_{1} \circ f\left(x_{0}^{+}\right) .
\end{aligned}
$$

Since $F$ and $g$ are strictly increasing, $f_{1}: I_{-} \rightarrow I_{+}$and $f_{2}: I_{+} \rightarrow I_{-}$are strictly decreasing, and $f$ is one-to-one, it follows from (3.17) that (3.10) holds. Thus, the given $f_{1}$, together with (3.10), (3.16), and (3.17), leads to (3.4). Refer to Figure 1. This completes the proof. $\square$

Theorem 2 Suppose that the usc multifunction (2.1) with $F\left(x_{0}\right)=[d, c]$ is one-to-one. If $\left.F\right|_{I_{-}}$and $\left.F\right|_{I_{+}}$are strictly increasing, then $F$ has nth iterative roots if and only if $F$ satisfies $F(b)<F(a)<c \leq x_{0}$ or $F(a)>F(b)>d \geq x_{0}$ or $F(b)<x_{0}<F(a)$.

(i) If $F(b)<F(a)<c \leq x_{0}$, then $F$ has a nonmonotonic nth iterative root defined by (3.1). Moreover, if $n$ is even and $\left.F\right|_{I_{-}}$has a regular fixed point, then $F$ also has a nonmonotonic

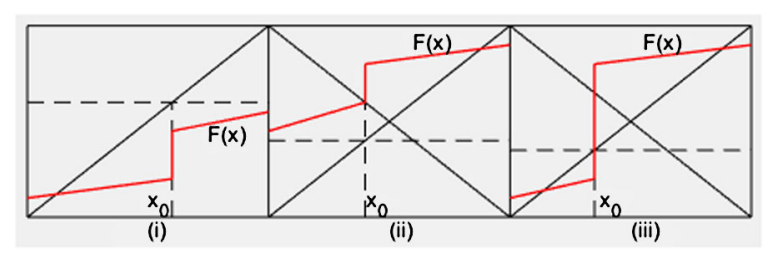

Figure 1 Three cases in Theorem 1. 
nth iterative root of the form (3.1), in which $f_{1}$ is a strictly decreasing function satisfying $f_{1}^{n}=\left.F\right|_{I_{-}}$.

(ii) If $F(a)>F(b)>d \geq x_{0}$, then $F$ has a nonmonotonic nth iterative root defined by (3.2). Moreover, if $n$ is even and $\left.F\right|_{I_{+}}$has a regular fixed point, then $F$ has also a nonmonotonic $n$th iterative root of the form (3.2), in which $f_{2}$ is a strictly decreasing function satisfying $f_{2}{ }^{n}=\left.F\right|_{I_{+}}$.

(iii) If $F(b)<x_{0}<F(a)$ and $n$ is odd, then $F$ has a nonmonotonic nth iterative root

$$
f(x)= \begin{cases}\left.F\right|_{I_{-}} \circ g^{-\frac{n-1}{2}}(x), & x \in I_{-}, \\ {\left[\left(\left.g\right|_{I_{-}}\right)^{-\frac{n-1}{2}}(d),\left(\left.g\right|_{I_{+}}\right)^{-\frac{n-1}{2}}(c)\right],} & x=x_{0}, \\ \left.F\right|_{I_{+}} \circ g^{-\frac{n-1}{2}}(x), & x \in I_{+},\end{cases}
$$

where $g$ is a strictly increasing $n$th iterative root of $F^{2}$. If $n$ is even, then $F$ has no iterative roots.

Proof The necessity directly comes from Lemma 2. In what follows, our attention is paid to the sufficiency.

Sufficiency. For case (i), we first construct a strictly increasing function (3.5) and a multifunction (3.7) as in case (i) of Theorem 1. Since $f_{1}$ is strictly increasing, from (3.8) and $c>d$ we have (3.10). Thus, (3.5), (3.7), and (3.10) yield a nonmonotonic $n$th iterative root (3.1) of $F$.

Assuming that $n$ is even and $\left.F\right|_{L_{-}}$has a regular fixed point, we can construct a strictly decreasing function $f_{1}$ and a multifunction (3.7) as in case (i) of Theorem 1. Since $f_{1}$ is strictly decreasing, (3.8) and $c>d$ lead to (3.9). Therefore, $f_{1}$ together with (3.7) and (3.9) gives a nonmonotonic $n$th iterative $\operatorname{root}(3.1)$ of $F$.

The proof of case (ii) is obtained from case (i) by the translation (3.11).

Case (iii). The assumption $F(b)<x_{0}<F(a)$ shows that

$$
F\left(I_{-}\right) \subset I_{+}, \quad F\left(I_{+}\right) \subset I_{-} .
$$

If $F$ has an $n$th iterative $\operatorname{root} f$, then, whether $n$ is odd or even, we assert that

$$
f\left(I_{-}\right) \subset I_{+}, \quad f\left(I_{+}\right) \subset I_{-} .
$$

In fact, suppose on the contrary that $f\left(I_{-}\right) \subset I_{-}$, whether $f\left(I_{+}\right) \subset I_{-}$or $f\left(I_{+}\right) \subset I_{+}$. Then we always have $F\left(I_{-}\right)=f^{n}\left(I_{-}\right) \subset I_{-}$, contrary to the first formula in (3.19). This implies that $f\left(I_{-}\right) \subset I_{+}$. The proof for $f\left(I_{+}\right) \subset I_{-}$is similar, and thus we have proved (3.20). It follows from (3.19) and (3.20) that

$$
\begin{aligned}
& \left.F\right|_{I_{-}}=\left(f_{1} \circ f_{2}\right)^{\frac{n-1}{2}} \circ f_{1}, \\
& \left.F\right|_{I_{+}}=\left(f_{2} \circ f_{1}\right)^{\frac{n-1}{2}} \circ f_{2},
\end{aligned}
$$

whence

$$
\begin{aligned}
& \left.F^{2}\right|_{I_{-}}=\left.\left.F\right|_{I_{+}} \circ F\right|_{I_{-}}=\left(f_{2} \circ f_{1}\right)^{n}, \\
& \left.F^{2}\right|_{I_{+}}=\left.\left.F\right|_{I_{-}} \circ F\right|_{I_{+}}=\left(f_{1} \circ f_{2}\right)^{n} .
\end{aligned}
$$


By Theorem 1(iii), (3.22) shows that there exists a strictly increasing $n$th iterative root $g$ of $F^{2}$ satisfying

$$
\left.g\right|_{I_{-}}=f_{2} \circ f_{1},\left.\quad g\right|_{I_{+}}=f_{1} \circ f_{2} .
$$

Substituting (3.23) into (3.21), we obtain

$$
\begin{aligned}
& f_{1}=\left.\left(\left.g\right|_{I_{+}}\right)^{-\frac{n-1}{2}} \circ F\right|_{I_{-}}, \\
& f_{2}=\left.\left(\left.g\right|_{I_{-}}\right)^{-\frac{n-1}{2}} \circ F\right|_{I_{+}},
\end{aligned}
$$

which implies that $f_{1}$ and $f_{2}$ are strictly increasing. Moreover, from (3.24) we have

$$
\begin{aligned}
& f_{1}\left(x_{0}^{-}\right)=\left.\left(\left.g\right|_{I_{+}}\right)^{-\frac{n-1}{2}} \circ F\right|_{I_{-}}\left(x_{0}{ }^{-}\right)=\left(\left.g\right|_{I_{+}}\right)^{-\frac{n-1}{2}}(c), \\
& f_{2}\left(x_{0}^{+}\right)=\left.\left(\left.g\right|_{I_{-}}\right)^{-\frac{n-1}{2}} \circ F\right|_{I_{+}}\left(x_{0}^{+}\right)=\left(\left.g\right|_{I_{-}}\right)^{-\frac{n-1}{2}}(d),
\end{aligned}
$$

implying $f_{1}\left(x_{0}{ }^{-}\right)>f_{2}\left(x_{0}{ }^{+}\right)$, that is, (3.10) holds. Thus, (3.24), (3.25), and (3.10) show that $F$ has a nonmonotonic $n$th iterative root (3.18).

If $n$ is even, then suppose on the contrary that $F$ has an $n$th iterative $\operatorname{root} f$. Since $g$ is a strictly increasing $n$th iterative root of $F^{2}$, we have

$$
\begin{aligned}
& F\left(I_{-}\right)=\left(\left.g\right|_{I_{-}}\right)^{\frac{n}{2}}\left(I_{-}\right)=\left(f_{2} \circ f_{1}\right)^{\frac{n}{2}} \subset I_{-}, \\
& F\left(I_{+}\right)=\left(\left.g\right|_{I_{+}}\right)^{\frac{n}{2}}\left(I_{+}\right)=\left(f_{1} \circ f_{2}\right)^{\frac{n}{2}} \subset I_{+},
\end{aligned}
$$

contrary to the fact that $F\left(I_{-}\right) \subset I_{+}$and $F\left(I_{+}\right) \subset I_{-}$. Refer to Figure 2. This completes the proof.

Theorem 3 Suppose that the usc multifunction (2.1) has $F\left(x_{0}\right)=[d, c]$. If $\left.F\right|_{I_{-}}$and $\left.F\right|_{I_{+}}$ are strictly decreasing, then $F$ has $n$th iterative roots if and only if $n$ is odd and $F$ satisfies $F(a)<x_{0}$ or $F(b)>x_{0}$ or $d \leq x_{0} \leq c$.

(i) If $F(a)<x_{0}$ and $n$ is odd, then $F$ has a strictly decreasing $n$th iterative root of the form (3.1), where $f_{1}$ is defined by

$$
f_{1}(x)= \begin{cases}\left.F\right|_{I_{-}} \circ \chi^{-\frac{n-1}{2}}(x), & a \leq x<x_{1}, \\ \chi^{\frac{n+1}{2}} \circ\left(\left.F\right|_{I_{-}}\right)^{-1}(x), & x_{1} \leq x<x_{0}\end{cases}
$$

in which $x_{1}$ is the unique fixed point of $\left.F\right|_{I_{-}}$, and $\chi$ is a strictly increasing $n$th iterative root of $\left(\left.F\right|_{I_{-}}\right)^{2}$.

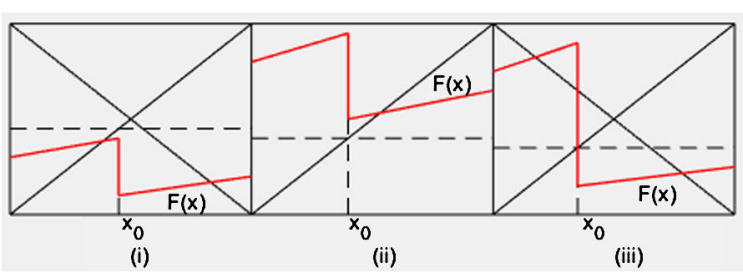

Figure 2 Three cases in Theorem 2. 
(ii) If $F(b)>x_{0}$ and $n$ is odd, then $F$ has a strictly decreasing $n$th iterative root of the form (3.2), where $f_{2}$ is defined by

$$
f_{2}(x)= \begin{cases}\left.F\right|_{I_{+}} \circ \psi^{-\frac{n-1}{2}}(x), & x_{0}<x \leq x_{2} \\ \psi^{\frac{n+1}{2}} \circ\left(\left.F\right|_{I_{+}}\right)^{-1}(x), & x_{2}<x \leq b\end{cases}
$$

in which $x_{2}$ is the unique fixed point of $\left.F\right|_{I_{+}}$, and $\psi$ is a strictly increasing $n$th iterative root of $\left(\left.F\right|_{I_{+}}\right)^{2}$.

(iii) If $d \leq x_{0} \leq c$ and $n$ is odd, then $F$ has a strictly decreasing $n$th iterative root

$$
f(x)= \begin{cases}\left.F\right|_{I_{-}} \circ \phi^{-\frac{n-1}{2}}(x), & x \in I_{-}, \\ {\left[\left(\left.\phi\right|_{I_{-}}\right)^{-\frac{n-1}{2}}(d),\left(\left.\phi\right|_{I_{+}}\right)^{-\frac{n-1}{2}}(c)\right],} & x=x_{0}, \\ \left.F\right|_{I_{+}} \circ \phi^{-\frac{n-1}{2}}(x), & x \in I_{+}\end{cases}
$$

where $\phi$ is a strictly increasing $n$th iterative root of $F^{2}$.

Proof Necessity. Suppose on the contrary that $n$ is even and $F$ has an $n$th iterative root $f$. Then only the following cases are possible:

Case 1. $f(I) \subset I_{-}$, or

Case 2. $f(I) \subset I_{+}$, or

Case 3. $f\left(I_{-}\right) \subset I_{-}, f\left(I_{+}\right) \subset I_{+}$, or

Case 4. $f\left(I_{-}\right) \subset I_{+}, f\left(I_{+}\right) \subset I_{-}$.

If either $f(I) \subset I_{-}$or $f(I) \subset I_{+}$, then it is clear that

$$
F(I)=f^{n}(I) \subset I_{+} \quad \text { or } \quad F(I)=f^{n}(I) \subset I_{-},
$$

contrary to the assumption on $F$. If $f\left(I_{-}\right) \subset I_{-}$and $f\left(I_{+}\right) \subset I_{+}$, then

$$
F\left(I_{-}\right)=f^{n}\left(I_{-}\right) \subset I_{-}, \quad F\left(I_{+}\right)=f^{n}\left(I_{+}\right) \subset I_{+},
$$

which also contradicts the assumption on $F$. If $f\left(I_{-}\right) \subset I_{+}$and $f\left(I_{+}\right) \subset I_{-}$, then as $n$ is even, we again get (3.29) and a contradiction. Thus, we have proved that $n$ is odd. The remainder directly comes from Lemma 2.

Sufficiency. Case (i). Note that

$$
\left.F\right|_{I_{-}}\left(\left[a, x_{1}\right)\right) \subset\left(x_{1}, x_{0}\right),\left.\quad F\right|_{I_{-}}\left(x_{1}, x_{0}\right) \subset\left[a, x_{1}\right)
$$

By Theorem 11.2.3 in [9] the strictly decreasing function $\left.F\right|_{I_{-}}$has a strictly decreasing $n$th iterative $\operatorname{root} f_{1}$ of the form (3.26). From (3.6) we still have (3.7), and, moreover, (3.8) and $d<c$ yield (3.10). Hence, (3.26), (3.7), and (3.10) show that $F$ has a strictly decreasing $n$th iterative $\operatorname{root} f$ of the form (3.1).

The proof of case (ii) is directly obtained from case (i) by the translation (3.11).

Case (iii). By the same argument as that of case (iii) in Theorem 2 we see that (3.21) still holds and there exists a strictly increasing $n$th iterative root $\phi$ of the strictly increasing 
multifunction $F^{2}$ satisfying

$$
\left.\phi\right|_{I_{-}}=f_{2} \circ f_{1},\left.\quad \phi\right|_{I_{+}}=f_{1} \circ f_{2} .
$$

Substituting (3.30) into (3.21), we obtain

$$
\begin{aligned}
& f_{1}=\left.\left(\left.\phi\right|_{I_{+}}\right)^{-\frac{n-1}{2}} \circ F\right|_{I_{-}}, \\
& f_{2}=\left.\left(\left.\phi\right|_{I_{-}}\right)^{-\frac{n-1}{2}} \circ F\right|_{I_{+}},
\end{aligned}
$$

which implies both $f_{1}$ and $f_{2}$ are strictly decreasing. Moreover, since $\phi$ is strictly increasing, from (3.31) we have

$$
\begin{aligned}
& f_{1}\left(x_{0}{ }^{-}\right)=\left.\left(\left.\phi\right|_{I_{+}}\right)^{-\frac{n-1}{2}} \circ F\right|_{I_{-}}\left(x_{0}{ }^{-}\right)=\left(\left.\phi\right|_{I_{+}}\right)^{-\frac{n-1}{2}}(c), \\
& f_{2}\left(x_{0}{ }^{+}\right)=\left.\left(\left.\phi\right|_{I_{-}}\right)^{-\frac{n-1}{2}} \circ F\right|_{I_{+}}\left(x_{0}{ }^{+}\right)=\left(\left.\phi\right|_{I_{-}}\right)^{-\frac{n-1}{2}}(d),
\end{aligned}
$$

implying (3.10). Thus, formulas (3.31), (3.32), and (3.10) show that $F$ has a strictly decreasing $n$th iterative root (3.28). Refer to Figure 3. This completes the proof.

Theorem 4 Suppose that the usc multifunction (2.1) with $F\left(x_{0}\right)=[c, d]$ is one-to-one, where $\left.F\right|_{I_{-}}$and $\left.F\right|_{I_{+}}$are strictly decreasing. Then $F$ has nth iterative roots if and only if $n$ is odd and $F$ satisfies $F(a)<F(b)<d \leq x_{0}$ or $F(b)>F(a)>c \geq x_{0}$ or $F(a)<x_{0}<F(b)$.

(i) If $F(a)<F(b)<d \leq x_{0}$ and $n$ is odd, then $F$ has a nonmonotonic nth iterative root of the form (3.1), where $f_{1}$ is defined by (3.26).

(ii) If $F(b)>F(a)>c \geq x_{0}$ and $n$ is odd, then $F$ has a nonmonotonic nth iterative root of the form (3.2), where $f_{2}$ is defined by (3.27).

(iii) If $F(a)<x_{0}<F(b)$ and $n$ is odd, then $F$ has a nonmonotonic nth iterative root of the form (3.3), where $f_{1}$ is defined by (3.26), and $f_{2}$ is defined by (3.27).

Proof The proof of necessity is similar to that of Theorem 3.

Sufficiency. Case (i). Using similar arguments as in the proof of case (i) of Theorem 3, we say that $\left.F\right|_{I_{-}}$has a strictly decreasing $n$th iterative $\operatorname{root} f_{1}$ of the form (3.26). Moreover, (3.7) comes from (3.6), and (3.9) comes from (3.8) and $c<d$. Thus, (3.26), (3.7), and (3.9) prove that $F$ has a nonmonotonic $n$th iterative root $f$ of the form (3.1).

The proof of case (ii) is directly obtained from case (i) by the translation (3.11).

Case (iii). The assumption on $F$ implies that $F\left(I_{-}\right) \subset I_{-}$and $F\left(I_{+}\right) \subset I_{+}$. Since $\left.F\right|_{I_{-}}$and $\left.F\right|_{I_{+}}$ are strictly decreasing, we can obtain their strictly decreasing $n$th iterative roots $f_{1}$ and $f_{2}$ defined by (3.26) and (3.27), respectively. Moreover, from (3.12) and $c<d$ we obtain (3.9).

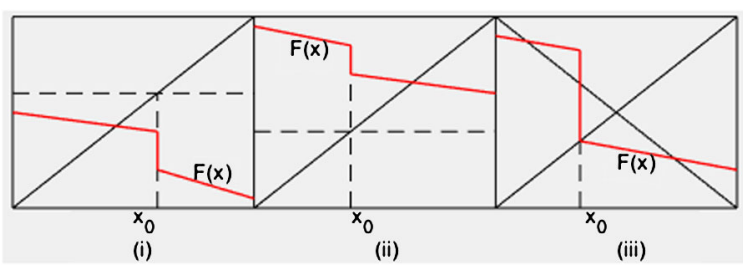

Figure 3 Three cases in Theorem 3. 


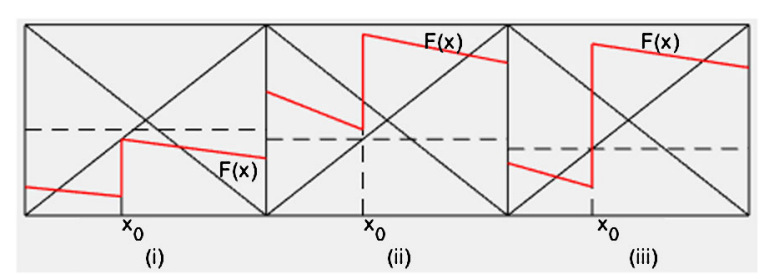

Figure 4 Three cases in Theorem 4.

Therefore, (3.26), (3.27), and (3.9) show that $F$ has a nonmonotonic $n$th iterative root of the form (3.3). Refer to Figure 4. This completes the proof.

\section{Examples}

In this section, we give examples. For convenience, to demonstrate our main results and avoid complicated computations, we only present a third iterative root of the given usc multifunctions.

Example 1 Consider the usc multifunction $\phi_{1}:[0,1] \rightarrow 2^{[0,1]}$ defined by

$$
\phi_{1}(x)= \begin{cases}\frac{27}{125} x+\frac{49}{250}, & x \in\left[0, \frac{1}{3}\right), \\ {\left[\frac{67}{250}, \frac{1761}{2000}\right],} & x=\frac{1}{3}, \\ \frac{27}{8000} x+\frac{1407}{1600}, & x \in\left(\frac{1}{3}, 1\right] .\end{cases}
$$

Clearly, both $\left.\phi_{1}\right|_{[0,1]_{-}}$and $\left.\phi_{1}\right|_{[0,1]_{+}}$are strictly increasing. Since $\phi_{1}\left(\frac{1}{3}^{-}\right)<\frac{1}{3}<\phi_{1}\left(\frac{1}{3}^{+}\right)$, in view of case (iii) of Theorem 1 , we say that $\phi_{1}$ has a strictly increasing $n$th iterative root (3.3). In fact, $\phi_{1}$ has a third iterative root as follows:

$$
\varphi_{1}(x)= \begin{cases}\frac{3}{5} x+\frac{1}{10}, & x \in\left[0, \frac{1}{3}\right), \\ {\left[\frac{3}{10}, \frac{4}{5}\right],} & x=\frac{1}{3}, \\ \frac{3}{20} x+\frac{3}{4}, & x \in\left(\frac{1}{3}, 1\right] .\end{cases}
$$

Example 2 Consider the usc multifunction $\phi_{2}:[0,1] \rightarrow 2^{[0,1]}$ defined by

$$
\phi_{2}(x)= \begin{cases}\frac{8}{125} x+\frac{87}{125}, & x \in\left[0, \frac{1}{2}\right) \\ {\left[\frac{43}{125}, \frac{91}{125}\right],} & x=\frac{1}{2}, \\ \frac{8}{125} x+\frac{6}{25}, & x \in\left(\frac{1}{2}, 1\right] .\end{cases}
$$

We see that $\left.\phi_{2}\right|_{[0,1]_{-}}$and $\left.\phi_{2}\right|_{[0,1]_{+}}$are strictly increasing. As $\phi_{2}(1)<\frac{1}{2}<\phi_{2}(0)$, using case (iii) of Theorem 2, $\phi_{2}$ has a nonmonotonic $n$th iterative root (3.18). Here, we give a third iterative root as follows:

$$
\varphi_{2}(x)= \begin{cases}\frac{2}{5} x+\frac{3}{5}, & x \in\left[0, \frac{1}{2}\right), \\ {\left[\frac{1}{5}, \frac{4}{5}\right],} & x=\frac{1}{2}, \\ \frac{2}{5} x, & x \in\left(\frac{1}{2}, 1\right] .\end{cases}
$$


Example 3 Consider the usc multifunction $\phi_{3}:[0,1] \rightarrow 2^{[0,1]}$ defined by

$$
\phi_{3}(x)= \begin{cases}-\frac{3}{320} x+\frac{467}{960}, & x \in\left[0, \frac{1}{3}\right), \\ {\left[\frac{29}{60}, \frac{185}{384}\right],} & x=\frac{1}{3}, \\ -\frac{1}{512} x+\frac{247}{512}, & x \in\left(\frac{1}{3}, 1\right] .\end{cases}
$$

Note that $\left.\phi_{3}\right|_{[0,1]_{-}}$and $\left.\phi_{3}\right|_{[0,1]_{+}}$are strictly decreasing and $\phi_{3}(1)>\frac{1}{3}$. From case (ii) of Theorem 3 we see that $\phi_{3}$ has a strictly decreasing $n$th iterative root of the form (3.2). We present a third iterative root by simple calculation:

$$
\varphi_{3}(x)= \begin{cases}-\frac{3}{5} x+\frac{4}{5}, & x \in\left[0, \frac{1}{3}\right), \\ {\left[\frac{1}{2}, \frac{3}{5}\right],} & x=\frac{1}{3}, \\ -\frac{1}{8} x+\frac{13}{24}, & x \in\left(\frac{1}{3}, 1\right] .\end{cases}
$$

Example 4 Consider the usc multifunction $\phi_{4}:[0,1] \rightarrow 2^{[0,1]}$ defined by

$$
\phi_{4}(x)= \begin{cases}-\frac{27}{1000} x+\frac{79}{125}, & x \in\left[0, \frac{2}{3}\right), \\ {\left[\frac{167}{500}, \frac{307}{500}\right],} & x=\frac{2}{3}, \\ -\frac{9}{500} x+\frac{173}{500}, & x \in\left(\frac{2}{3}, 1\right] .\end{cases}
$$

Note that $\left.\phi_{4}\right|_{[0,1]_{-}}$and $\left.\phi_{4}\right|_{[0,1]_{+}}$are strictly decreasing and $\phi_{4}(0)<\phi_{4}(1)<\phi_{4}\left(\frac{2}{3}^{+}\right)<\frac{2}{3}$. In view of case (i) of Theorem $4, \phi_{4}$ has a nonmonotonic $n$th iterative root of the form (3.1). Here, a nonmonotone third iterative root of $\phi_{4}$ is given as

$$
\varphi_{4}(x)= \begin{cases}-\frac{3}{10} x+\frac{2}{5}, & x \in\left[0, \frac{2}{3}\right), \\ {\left[\frac{1}{5}, \frac{3}{5}\right],} & x=\frac{2}{3}, \\ -\frac{1}{5} x+\frac{11}{15}, & x \in\left(\frac{2}{3}, 1\right] .\end{cases}
$$

\section{Acknowledgements}

The authors would like to thank the referees for their valuable comments and suggestions. This work is supported by Shandong Provincial Natural Science Foundation (ZR2017MA019, ZR2014AL003) and Scientific Research Fund of Binzhou University (BZXYL1703).

\section{Competing interests}

The authors declare that they have no competing interests.

\section{Authors' contributions}

The authors completed the paper together. They also read and approved the final manuscript.

\section{Publisher's Note}

Springer Nature remains neutral with regard to jurisdictional claims in published maps and institutional affiliations.

Received: 14 January 2017 Accepted: 20 October 2017 Published online: 25 November 2017

\section{References}

1. Li, L, Jarczyk, J, Jarczyk, W, Zhang, W: Iterative roots of mappings with a unique set-value point. Publ. Math. (Debr.) 75, 203-220 (2009)

2. Babbage, C: Essay towards the calculus of functions. Philos. Trans. R. Soc. Lond. 105, 389-423 (1815)

3. Baron, $K$, Jarczyk, W: Recent results on functional equations in a single variable, perspectives and open problems. Aequ. Math. 61, 1-48 (2001)

4. Jarczyk, W: Recent results on iterative roots. ESAIM Proc. Surv. 46, 47-62 (2014) 
5. Targonski, G: New directions and open problems in iteration theory. Grazer Math. Ber. 229 (1984)

6. Targonski, G: Progress of iteration theory. Aequ. Math. 50, 50-72 (1995)

7. Zhang, W: PM functions, their characteristic intervals and iterative roots. Ann. Pol. Math. 65, 119-128 (1997)

8. Kuczma, M: Functional Equations in a Single Variable. Monografie Mat., vol. 46. Polish Sci., Warszawa (1968)

9. Kuczma, M, Choczewski, B, Ger, R: Iterative Functional Equations. Cambridge University Press, Cambridge (1990)

10. Targonski, G: Topics in Iteration Theory. Vandenhoeck \& Ruprecht, Gottingen (1981)

11. Bödewadt, UT: Zur Iteration reeller Funktionen. Math. Z. 49, 497-516 (1944)

12. Hădukov, Pl: On searching a function from a given iterate. Uč. Zap. Buriatsk. Ped. Inst. 15, 3-28 (1958) (in Russian)

13. Kuczma, M: On the functional equations $\varphi^{n}(x)=g(x)$. Ann. Pol. Math. 11, 161-175 (1961)

14. Zhang, J, Yang, L: Discussion on iterative roots of piecewise monotone functions. Acta Math. Sin. 26, 398-412 (1983) (in Chinese)

15. Zhang, J, Yang, L, Zhang, W: Some advances on functional equations. Adv. Math. 26, 385-405 (1995)

16. Li, L, Yang, D, Zhang, W: A note on iterative roots of PM functions. J. Math. Anal. Appl. 341, 1482-1486 (2008)

17. Liu, L, Jarczyk, W, Li, L, Zhang, W: Iterative roots of piecewise monotonic functions of nonmonotonicity height not less than 2. Nonlinear Anal. 75, 286-303 (2012)

18. Liu, L, Zhang, W: Non-monotonic iterative roots extended from characteristic intervals. J. Math. Anal. Appl. 378, 359-373 (2011)

19. Li, L, Chen, J: Iterative roots of piecewise monotonic functions with finite nonmonotonicity height. J. Math. Anal. Appl. 411(1), 395-404 (2014)

20. Humke, PD, Laczkovich, M: The Borel structure of iterates of continuous functions. Proc. Edinb. Math. Soc. 32(2), 483-493 (1989)

21. Simon, K: Some dual statements concerning Wiener measure and Baire category. Proc. Am. Math. Soc. 106, 455-463 (1989)

22. Blokh, AM: The set of all iterates is nowhere dense in $C([0,1],[0,1])$. Trans. Am. Math. Soc. 333, 787-798 (1992)

23. Targonski, G: Phantom iterates of continuous functions. In: Iteration Theory and Its Functional Equations, Lochau, 1984. Lecture Notes in Math., vol. 1163, pp. 196-202. Springer, Berlin (1985)

24. Powierża, T: Strong set-valued iterative roots. Grazer Math. Ber. 344, 51-56 (2001)

25. Powierża, T: Higher order set-valued iterative roots of bijections. Publ. Math. (Debr.) 61, 315-324 (2002)

26. Jarczyk, W, Powierża, T: On the smallest set-valued iterative roots of bijections. Int. J. Bifurc. Chaos Appl. Sci. Eng. 13, 1889-1893 (2003)

27. Jarczyk, W, Zhang, W: Also multifunctions do not like iterative roots. Elem. Math. 62(2), $73-80$ (2007)

\section{Submit your manuscript to a SpringerOpen ${ }^{\circ}$ journal and benefit from:}

- Convenient online submission

- Rigorous peer review

- Open access: articles freely available online

- High visibility within the field

- Retaining the copyright to your article

Submit your next manuscript at $\gg$ springeropen.com 